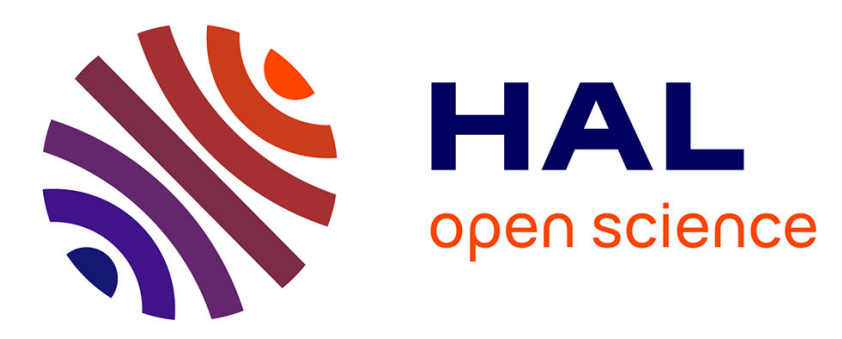

\title{
Fast Image-Based Mitral Valve Simulation from Individualized Geometry
}

Pierre-Frédéric Villard, Peter E. Hammer, Douglas P. Perrin, Pedro J. del Nido, Robert Howe

\section{- To cite this version:}

Pierre-Frédéric Villard, Peter E. Hammer, Douglas P. Perrin, Pedro J. del Nido, Robert Howe. Fast Image-Based Mitral Valve Simulation from Individualized Geometry. The International Journal of Medical Robotics and Computer Assisted Surgery, 2018, 14 (2), pp.e1880. 10.1002/rcs.1880 . hal01643868

\section{HAL Id: hal-01643868 \\ https://hal.science/hal-01643868}

Submitted on 25 Jan 2018

HAL is a multi-disciplinary open access archive for the deposit and dissemination of scientific research documents, whether they are published or not. The documents may come from teaching and research institutions in France or abroad, or from public or private research centers.
L'archive ouverte pluridisciplinaire HAL, est destinée au dépôt et à la diffusion de documents scientifiques de niveau recherche, publiés ou non, émanant des établissements d'enseignement et de recherche français ou étrangers, des laboratoires publics ou privés. 


\title{
Fast Image-Based Mitral Valve Simulation from Individualized Geometry
}

\author{
Pierre-Frederic Villard ${ }^{1,2 *}$, Peter E. Hammer ${ }^{2,3}$, Douglas P. Perrin ${ }^{2,3}$, Pedro J. del Nido ${ }^{3}$, \\ Robert D. Howe ${ }^{2}$ \\ ${ }^{1}$ LORIA, University of Lorraine, Inria, Nancy, France \\ ${ }^{2}$ Harvard School of Engineering and Applied Sciences, Cambridge, MA, USA \\ ${ }^{3}$ Department of Cardiac Surgery, Boston Children's Hospital, Boston, MA, USA
}

\begin{abstract}
SUMMARY
Background: Common surgical procedures on the mitral valve of the heart include modifications to the chordae tendineae. Such interventions are used when there is extensive leaflet prolapse caused by chordae rupture or elongation. Understanding the role of individual chordae tendineae before operating could be helpful to predict if the mitral valve will be competent at peak systole. Biomechanical modeling and simulation can achieve this goal.

Methods: We present a method to semi-automatically build a mitral valve computational model from micro CT (computed tomography) scans: after manually picking chordae fiducial points, the leaflets are segmented and the boundary conditions as well as the loading conditions are automatically defined. Fast Finite Element Method (FEM) simulation is carried out using Simulation Open Framework Architecture (SOFA) to reproduce leaflet closure at peak systole. We develop three metrics to evaluate simulation results: i) point-to-surface error with the ground truth reference extracted from the CT image, ii) coaptation surface area of the leaflets and iii) an indication if the simulated closed leaflets leak.

Results: We validate our method on three explanted porcine hearts and show that our model predicts the closed valve surface with point-to-surface error of appoximately $1 \mathrm{~mm}$, a reasonable coaptation surface area, and absence of leak at peak systole (maximum closed pressure). We also evaluate the sensitivity of our model to changes in various parameters (tissue elasticity, mesh accuracy, and the transformation matrix used for CT scan registration). We also measure the influence of the chordae tendineae positions on simulation results and show that marginal chordae have a greater influence on the final shape than intermediate chordae. Conclusions: The mitral valve simulation can help the surgeon understand valve behaviour and anticipate the outcome of a procedure.
\end{abstract}

KEY WORDS: Mitral valve model, FEM simulation, image-based model, validation metrics

\section{INTRODUCTION}

The mitral valve is located in the left chamber of the heart. Its role consists of alternately opening so the blood can flow from the left atrium to the left ventricle and closing so the blood can flow

${ }^{*}$ Correspondence to: Pierre-Frederic Villard, LORIA, University of Lorraine, Nancy, France. E-mail: pierrefrederic.villard@loria.fr 
from the left ventricle to the aorta through the aortic valve without going back into the left atrium. Valve function results from the interaction of various anatomical parts (Fig.1 left): i) two leaflets (anterior and posterior) that open and close, ii) the chordae tendineae that tether the leaflets to maintain valve shape during closure (systole), iii) the papillary muscles which anchor the chordae to the ventricle wall and pull down during closure, and iv) the annulus around which the leaflets attach circumferentially to the left ventricle.

Common mitral valve surgical procedures include modifications to the chordae. Such interventions are used when there is extensive prolapse of a leaflet caused by chordae rupture or elongation. This prolapse often allows backflow into the left atrium and must be treated. Chordae modification procedures include: i) secondary transposition from the posterior leaflet (Fig.1 right.a), ii) chordae transposition from the posterior leaflet (Fig.1 right.b), iii) chordae replacement using artificial chordae in the case of chordae rupture (Fig.1 right.c) and iv) chordae shortening in the case of chordae elongation (see Fig.1 right.d) [1].

Understanding the effect of particular chordae on valve competence would significantly help cardiologists and surgeons preoperatively form a plan for valve surgical repair. Biomechanical modeling and simulation can inform this process. Many research teams have worked on this problem [2]. In these studies, the common strategy is to use a generic, rather than a subject-specific, model of valve geometry. Recently several image-based models have been proposed [3, 4, 5, 6]. However, in these models, chordae are still based on generic models and the FEM (finite element method) -based computation engines that are used are not efficient enough to be used in the clinical workflow.

Our strategy is to build a complete semi-automatic pipeline to construct an image-based FEM model of the mitral valve simulating leaflet closure at peak systole. We are targeting a framework that can robustly work with geometries extracted from clinical data. We are starting with micro CT images, but other image modalities will be considered in the future. We construct the computational model from real subject geometries with image processing. Our aim is also to do real-time simulation that can be used in the clinical workflow. Finally, we validate our simulations on real cases of valve closure in order to simulate the real shapes of the leaflets and study the influence of the chordae attachment points.

Many studies have already been conducted on simulation of the heart valves (See comparison on Table I). Several research groups have developed 3D FEM models of the mitral valve. The workflow is often similar, including these steps: i) extracting the valve anatomy from medical imaging, ii) defining the boundary conditions and the loading and iii) doing an FEM analysis applying mechanical test data based on assumptions. Below is a review of some of these studies.

Kunzelman et al. [7, 8, 9] extracted geometry from explanted porcine hearts. The leaflet was considered free to rotate at the annulus, and the chords were attached at both papillary muscles and leaflet edges with free rotation. In systole, annular contraction was modeled by applying forces, and papillary muscles were moved down $1 \mathrm{~mm}$ in the apical direction. A linear, anisotropic material model was used to account for the presence of a preferred material direction. In their results, they analyzed leaflet and chordae stresses in response to modifications in the valve configuration (papillary muscle displacement, annulus dilation, etc.).

Sacks et al. [10] extracted the mitral valve anatomy based on porcine heart micro CT scans as well. The pressure on the leaflet was given by a time-dependent physiological curve, and the displacements of the annulus and papillary muscles were given by sonomicrometry transducers 


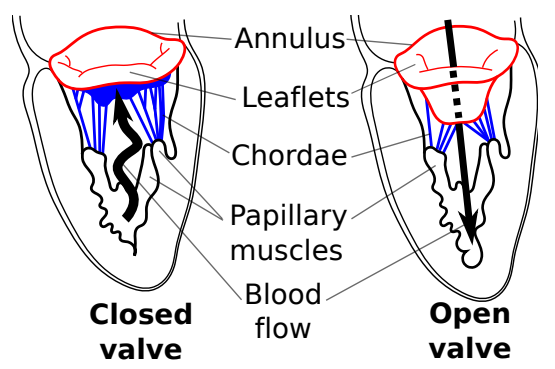

a)

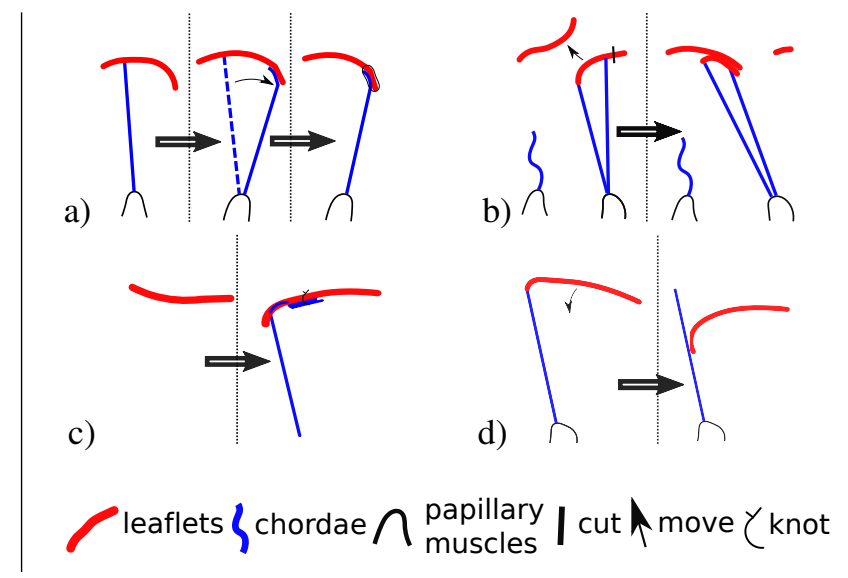

c)

Figure 1. Mitral valve anatomy and surgical operations on chordae. left Appearance of the closed valve (during systole) and open valve (during diastole), right Surgical procedures on chordae tendineae: a) secondary transposition from the posterior leaflet, b) chordae transposition from the posterior leaflet, c) chordae replacement using artificial chordae and d) chordae shortening in the case of chordae elongation.

[11]. They used a tension-only model for the chordae. An experimentally-derived hyperelastic law was used to model the leaflets. In their results, they analyzed the leaflet stresses to show that valve failure mechanisms occur at abnormally high stresses and that higher stresses occur at peak systolic loading.

In Prot et al. [12], the geometry was extracted from a porcine heart that had been dissected and photographed. The leaflets and chordae were reconstructed. The papillary muscles were assumed to be fixed. There was a self-contact condition between the two layers of the leaflet. The annulus displacement was measured with ultrasound before dissection while under the same pressure that was later applied in the computational model. The leaflet constitutive law was derived from the hyperelastic law proposed by [13]. The leaflets were meshed with triangles and the chordae by trusses. In their results, they compared the leaflet stress distribution and chordae tensions with values from the literature.

In Wang et al. [6], the authors extracted the valve anatomy from a multi-slice CT scan of a human subject. The geometry included the mitral valve leaflets, the papillary muscles and 21 chordae. The displacements of both the annulus and the chordae attachments on the papillary muscles were tracked across a sequence of CT acquisitions and applied as imposed displacements. A timedependent pressure based on the cardiac cycle was applied to the leaflet. An anisotropic hyperelastic Ogden model was used to model the chordae. 3D elements were used to capture the mitral valve and 1D elements for the chordae. In their results, they compared model stress and strain predictions with values from the literature. They also measured coaptation characteristics to mimic clinical assessent.

Hammer et al. [14] developed a mass-spring framework to simulate mitral valve closure using a nonlinear anisotropic constitutive law. Chordae were indirectly taken into account by constraining various displacements of leaflet vertices based on their location. In [15], the previous model was adapted to perform real-time with haptic feedback. In their results, they computed the surface-tosurface error between simulation and measured data.

More recently, Toma et al [16] developed a mitral valve computational model from the ovine heart. They focused on one dataset using an accurate mesh with an anisotropic constitutive law. 
Table I. Summary of the state of the art for mitral valve modeling

\begin{tabular}{|c|c|c|c|c|c|c|}
\hline & $\begin{array}{l}\text { Model } \\
\text { geometry }\end{array}$ & $\begin{array}{l}\text { Leaflet } \\
\text { model }\end{array}$ & $\begin{array}{l}\text { Anisotropy } \\
\text { \& hyper- } \\
\text { elasticity }\end{array}$ & $\begin{array}{c}\text { Chordae } \\
\text { model }\end{array}$ & $\begin{array}{l}\text { Comp- } \\
\text { uting } \\
\text { time }\end{array}$ & Metrics \\
\hline $\begin{array}{c}\text { Kunzelman } \\
\text { et al.[8,9] }\end{array}$ & $\begin{array}{l}\text { Manual } \\
\text { Generic }\end{array}$ & $\begin{array}{l}\text { Shell } \\
\text { FEM }\end{array}$ & Yes & $\begin{array}{l}\text { Truss } \\
\text { FEM }\end{array}$ & na & $\begin{array}{c}\text { Stress } \\
\text { analysis }\end{array}$ \\
\hline $\begin{array}{c}\text { Sacks } \\
\text { et al.[10] }\end{array}$ & $\begin{array}{l}\text { Manual } \\
\text { Generic }\end{array}$ & $\begin{array}{l}\text { Shell } \\
\text { FEM }\end{array}$ & Yes & $\begin{array}{l}\text { Truss } \\
\text { FEM }\end{array}$ & na & $\begin{array}{c}\text { Stress } \\
\text { analysis }\end{array}$ \\
\hline $\begin{array}{c}\text { Prot } \\
\text { et al. [12] }\end{array}$ & $\begin{array}{l}\text { Manual } \\
\text { Generic }\end{array}$ & $\begin{array}{l}\text { Shell } \\
\text { FEM }\end{array}$ & Yes & $\begin{array}{l}\text { Truss } \\
\text { FEM }\end{array}$ & na & $\begin{array}{c}\text { Stress } \\
\text { analysis }\end{array}$ \\
\hline $\begin{array}{c}\text { Wang } \\
\text { et al. [6] }\end{array}$ & $\begin{array}{c}\text { Manual } \\
\text { Image-based }\end{array}$ & $\begin{array}{l}\text { Shell } \\
\text { FEM }\end{array}$ & Yes & $\begin{array}{l}\text { Truss } \\
\text { Fem }\end{array}$ & na & $\begin{array}{c}\text { Error distance } \\
\text { Coaptation }\end{array}$ \\
\hline $\begin{array}{l}\text { Hammer } \\
\text { et al. [14] }\end{array}$ & $\begin{array}{c}\text { Manual } \\
\text { Image-based }\end{array}$ & $\begin{array}{l}\text { Mass- } \\
\text { spring }\end{array}$ & Yes & $\begin{array}{l}\text { Tension } \\
\text {-only rod }\end{array}$ & $20 \mathrm{sec}$ & $\begin{array}{c}\text { Error distance } \\
\text { Coaptation }\end{array}$ \\
\hline $\begin{array}{c}\text { Toma } \\
\text { et al. [16] }\end{array}$ & $\begin{array}{l}\text { Semi-automatic } \\
\text { Image-based }\end{array}$ & $\begin{array}{l}\text { Tetrahedra } \\
\text { FEM }\end{array}$ & Yes & $\begin{array}{c}\text { Tetrahedra } \\
\text { FEM }\end{array}$ & na & $\begin{array}{c}\text { Stress } \\
\text { analysis }\end{array}$ \\
\hline $\begin{array}{l}\text { This } \\
\text { study }\end{array}$ & $\begin{array}{l}\text { Semi-automatic } \\
\text { Image-based }\end{array}$ & $\begin{array}{c}\text { Triangle } \\
\text { FEM }\end{array}$ & No & $\begin{array}{l}\text { Mass- } \\
\text { spring }\end{array}$ & $15 \mathrm{sec}$ & $\begin{array}{l}\text { Error distance } \\
\text { Coaptation } \\
\text { Leak }\end{array}$ \\
\hline
\end{tabular}

They also took into account blood flow in the boundary conditions. As a result, they analyzed the stress distribution as well as the chordal forces at the attachement points on papillary muscles to compare them with in vitro results.

The aforementioned research is based either on tedious preliminary work of manual segmentation or over-simplified parametric geometries. In this paper, we propose to automatically build a computational model of the mitral valve with minor manual inputs. Our aim is also to do realtime simulation that can be used in the clinical workflow while being able to reproduce real closed leaflet shapes at peak systole.

\section{MATERIALS AND METHODS}

Here we describe the methods used to create a subject-specific model of the mitral valve. First we describe image acquisition followed by image segmentation. We then describe how the major components of the valve are modeled and how the closure of the valve at peak systolic pressure is simulated and evaluated.

\subsection{Image acquisition}

In this study we used porcine hearts as they have been shown to be a good proxy for human hearts [7]. The image-based model was extracted from three fresh isolated hearts from $30-40 \mathrm{~kg}$ female Yorkshire pigs imaged using high resolution computed tomography (MicroCAT, Siemens, Munich, Germany). For each subject, the heart was excised, and images of the mitral valve in two states were acquired: one with no pressure to have an unstressed valve state and one with $120 \mathrm{mmHg}$ of air pressure delivered through the aorta to close the mitral valve and statically load it. A protocol was 
established to ensure high quality $\mu \mathrm{CT}$ scans and included carefully supporting the heart (Fig. 2.a) and pressurizing with air (Fig. 2.b).

a)

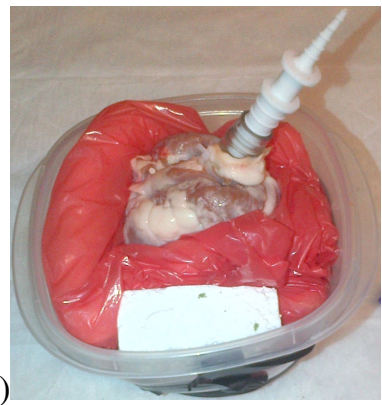

b)

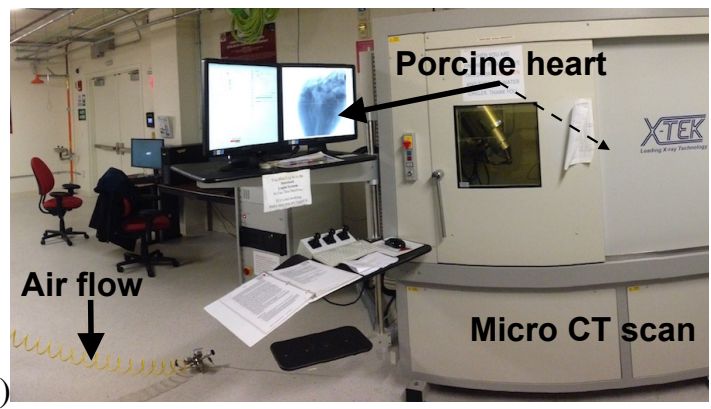

Figure 2. a) Foam system to support the heart. b) $\mu \mathrm{CT}$ scanner containing a heart pressurized using a regulated air supply.

Scanner settings were established based on trial-and-error in order to avoid artifact due to movement or drying/shrinking of the cardiac tissues during image acquisition. The number of scanning angles was 1600 , the exposure time for each image was $500 \mathrm{~ms}$, the $\mathrm{x}$-ray tube power was set to $10.5 \mathrm{~W}$ with $\mathrm{U}=175 \mathrm{kV}$, and a $0.5 \mathrm{~mm}$ copper filter was used to achieve sufficient contrast and resolution.

The result after reconstruction was a $2000 \times 2000 \times 2000$ voxel 3D image with isotropic voxel resolution of $0.1 \mathrm{~mm}$ and an 8-bit integer format from which a region of interest (ROI) for the mitral valve was selected. These scans produced high contrast volumetric images mainly composed of air (Hounsfield density $\approx 1000$ ) and soft tissue (Hounsfield density $\in[-100,300]$ ).

For each subject, the heart was excised, and images of the mitral valve in two states were acquired: one with no pressure to have an unstressed valve state and one with $120 \mathrm{mmHg}$ of air pressure delivered through the aorta to close the mitral valve and statically load it. In the following sections of the article these two states are respectively referred to as initial and final configurations.

\subsection{Image segmentation}
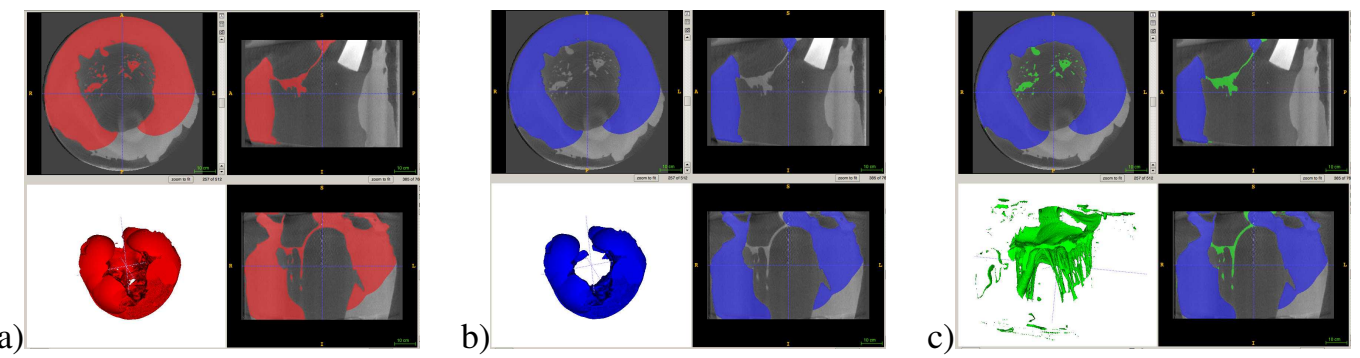

Figure 3. Level-set segmentation results. a) Force allowing small curvatures . b) Force only allowing high curvatures. c) Boolean difference

A method to semi-automatically segment the leaflets and chordae was developed and performed on the micro CT data. It uses the level-set segmentation framework described in [17] which consists of a three stage process: computing the active contour image, placing spherical seeds on the same 

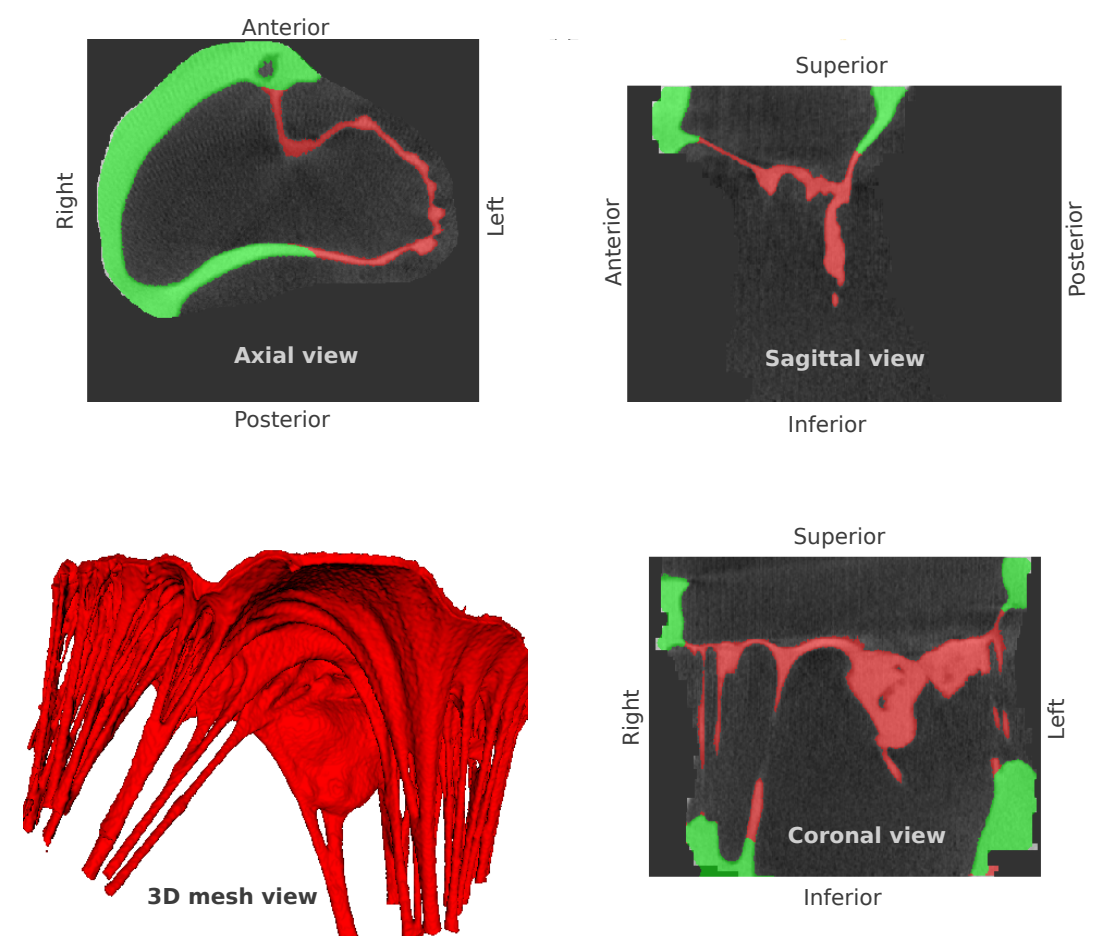

Figure 4. Micro CT scan with segmentation results on axial, sagittal, coronal and 3D mesh view. Green: ventricle wall, red: mitral valve.

image, and running an iterative evolution function. Two labeled images are computed using only one contour image and manually placing (only once) seeds on the ventricular endocardium. The active contour used the 'Region Competition' method [18], and it computes a probability map. The evolution function is based on a curvature term weighted by $\alpha$ with $\alpha \in[0,1]$ and a gradient intensity based force [19]. The first labeled image utilized a small curvature weight for the contour evolution differential equation, thus allowing the region-growing algorithm to capture thin structures such as the leaflets and the chordae (see Fig.3.a). The second labeled image uses a high curvature weight producing a smoother contour boundary while ignoring the leaflets and the chordae. The region cannot grow in the thin structures (Fig.3.b).

The boolean difference between both of these images (Fig.3.a and 3.b) gives the final leafletchordae set (see Fig.3.c). Fig. 4 shows the same information after having extracted the largest connected component [20].

\subsection{Image registration}

Micro CT scans for both initial (unpressurezed) and final (pressurized) configurations were acquired at different times. Between these two scans, the position of the valve changed. The first step was therefore to register the two configurations. A rigid registration method [21] was used to match the corresponding meshes in initial and final configurations. To ensure consistency, registration was initialized using four reference points located on the anterior and posterior border of the leaflets and identified on both configurations. More precisely, if the $\mathrm{Z}$ projection of the valve forms an ellipse, the four points are placed where its minor and major axes intersect the ellipse. 


\subsection{Modeling the valve leaflets}

In order to simulate valve closure, an initial mesh geometry with boundary and loading conditions is necessary. The final shape of the leaflets (at peak systole) is also needed for validation purposes. Meshes of both states of the leaflets were generated from the segmented 3D images using the marching cubes algorithm. The meshes were decimated using a fast and automatic method where the only input is the number of final points comprising the mesh ([22]). For more information about the software see http://alice.loria.fr/index.php/erc-vorpaline.html.

The whole valve was aligned with the z-axis (i.e., so that the best-fit plane to the annulus is normal to the z-direction) using a transformation matrix consisting of two pure rotations (Fig. 5). The purpose of reorientating the valve is to correct any slight misalignment in order to improve the robustness of our algorithms. It is twofold. First, it allows the top surface of the valve to be extracted by comparing the surface normals with the $\mathbf{Z}$ direction. Second, it is used to filter the chordae modeled by line segments as detailed in the next section. A one-layer surface was extracted from the valve by comparing the surface normals with the $\mathbf{Z}$ direction and taking the largest connected component. The top surface is the mesh of the leaflets.

The result is a mesh of the leaflets. Fig. 5. $a_{1}$ shows an initial mesh from our previous process in its original orientation. Fig. 5. $a_{2}$ shows the result of our leaflet geometry extraction with a reorientation to the $\mathbf{Z}$ axis and with only one layer to define the mesh.

\subsection{Modeling the chordae}

Chordae are flexible linear elements supporting only tension and are commonly modeled as a branching network of 1D elements (see $\S 1$ ). Each chord is modeled as an insertion point on the leaflet, an attachment point on the papillary muscle, and branch points. This section details, in three steps, how we build the chordae geometrical model used in the simulation.

2.5.1. Extracting chordae central lines from CT scan voxels In the context of this study, we manually picked the chordae for each pig subject on the unstressed state and we also measured their taught length in the pressurized state. The two meshes (with and without pressure) of the leaflets and the information on the chordae are the input data to an automatic pipeline defining the biomechanical model.

2.5.2. Extracting chordae structure: insertion and branch points Papillary muscles contract while the apex of the heart moves toward the annulus. It is commonly assumed that this motion is fully compensatory and that the chordae can be assumed to be fixed at the papillary muscles [12]. Similarly the other chordae extremities are attached to the leaflets.

Since the valve was reoriented in the $\mathbf{Z}$ direction, all the chordae can be sorted (See Fig. 5.b) using each point $(P) \mathbf{Z}$-coordinate $\left(Z_{P}\right)$ so that if $\mathbf{A}$ and $\mathbf{B}$ are the two set of points of every line segments defining a chord, the list of $\mathbf{A B}$ line segments is defined by

$$
\text { if } Z_{A}>Z_{B} \text { then switch } \mathbf{A} \text { and } \mathbf{B}
$$

The set of points belonging to the chordae line segments $\left(\mathbf{C}_{\text {Leaf }}\right)$ attached to the leaflets (whose set of vertices is $\mathbf{V}_{\text {Leaf }}$ ) is defined by 

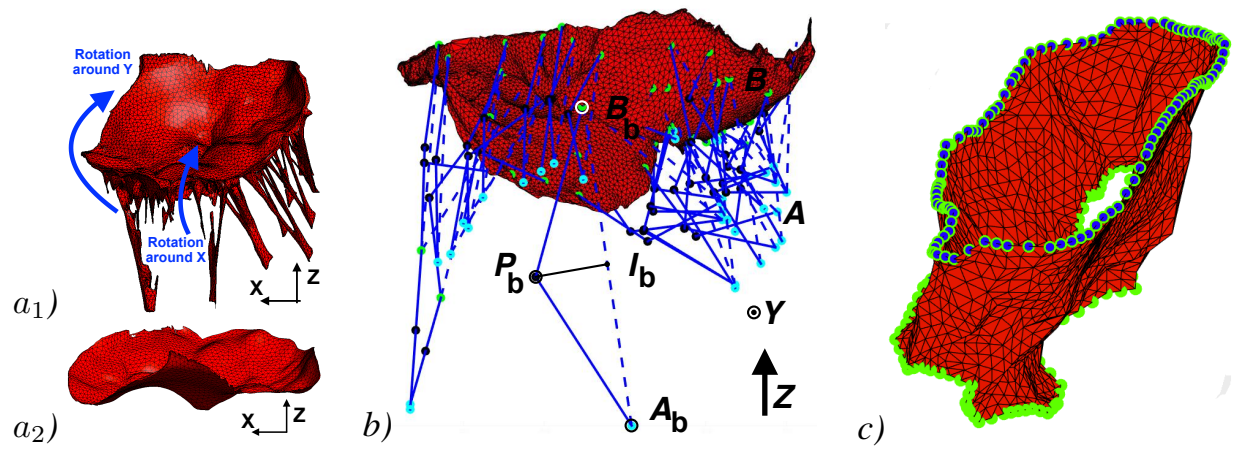

Figure 5. Data pre-processing. $a$ ) Leaflet re-orientation to to extract a one-layer surface. $b$ ) Chordae flexure and attachment points. Green: points attached to the leaflets, cyan: points attached to the papillary muscles, black: branch points, black: points added for flexure, blue dashed line: line segments before flexure. c) Annulus points. Green: vertices belonging to the leaflets contours, blue: vertices belonging to the annulus.

$$
\mathbf{C}_{\text {Leaf }}=\min \left(\operatorname{dist}\left(\mathbf{V}_{\text {Leaf }}, \mathbf{B}\right)\right)
$$

The set of points $\mathbf{C}_{p a p}$ attached to the papillary muscles is defined by $\mathbf{C}_{p a p}=A \cap B$.

At this stage, the structure of the chordae architecture is known for the valve pressurized configuration. It is defined by lines with vertices that could be either insertion points $\left(C_{\text {Leaf }}\right.$ or $\left.C_{\text {pap }}\right)$ or branch points. The next step consists in using them to build a chordae geometry configuration when the valve is open.

\subsubsection{Extracting configuration of the chordae in the valve unstressed state As previously} mentioned, the unstressed leaflets were directly extracted from the micro CT scan, but only the insertion points of the chordae are known exactly since they are slack (unstressed). The only information measured with certainty is their maximal length during peak systole. Their tensile stiffness is known to be very high [9]. Our strategy was then to let each chordae segment articulate about one joint rather than buckle, and behavior of the chordae network was simulated as a stiff mass-spring system. The articulation is needed because the initial condition of the valve closure simulation corresponds to an unstressed state. This means the leaflets must be open and the chordae must be attached from the papillary muscles to the correct location on the leaflet. Instead of reproducing chordae buckling via a continuous curved path between segment endpoints, a simple division into two subsegments was developed. The chordae behavior will range from a folded state to a fully stretched state during systole (see Fig. 5.a). By applying basic geometry and defining a direction of folding, we can define the exact degree of folding.

The aim is to compute the set of articulation points $P_{b}$. A point $I_{b}$ is computed in the middle of the last line segment of each chordae $A_{b} B_{b}$. The position of $P_{b}$ corresponding to the articulation point of line segment $A_{b} B_{b}$ is then given by Eq.(3). $P_{b}$ is the construction point that allows to build the chordae flexure geometry. The final chordae position will be a straight line so the articulation can occur anywhere in the $X Y$ plane. We arbitrarily let $Y$ be the direction of flexure. $A_{I}$ and $B_{I}$ are the initial positions of points $A$ and $B$, respectively, in the open valve configuration. 


$$
\begin{aligned}
I b & =\left(A_{b}+B_{b}\right) / 2 \\
F l & =A_{b} I_{b} /\left\|A_{b} I_{b}\right\| \times Y \\
\left\|I_{b} P_{b}\right\|^{2}+\left\|A_{b} I_{b}\right\|^{2} & =\left\|A_{I} B_{I} / 2\right\|^{2} \\
I_{b} P_{b} & =F l\left\|I_{b} P_{b}\right\|
\end{aligned}
$$

The output is the geometrical information of the chordae tree structure during the unstressed valve configuration (solid blue lines on Fig. 5.b).

\subsection{Modeling the valve annulus}

During image acquisition, the annulus moves from an initial position (not pressurized) to a final position (pressurized). Both are given by the contour of the leaflets. The edge of the surface mesh is identified using connectivity, which defines the annulus. The vertices belonging to these edges are the ones constituting the annulus. It is slightly more complicated for the initial position as the leaflets are open: the connectivity in this case is a list of points containing both the annulus and the extremity of the leaflet. However since the final positions of the annulus are now known, only the points belonging to the previous edge-based sort that are close enough to the final annulus position are kept. Results of this algorithm are illustrated in Fig.5.b).

As both micro CT scans were acquired at different locations, it was necessary to register them. We used the valve mesh data and the iterative closest point algorithm guided by four initial points on each geometry to ensure rigid-registration consistency. While the horizontal alignment was consistent because both leaflets were properly centered at the end of the algorithm, the vertical alignment was slightly inaccurate and will later be optimized.

\subsection{Simulating the valve at peak systole}

We simulate mitral valve closure using the SOFA framework [23]. SOFA is an open-source library targeting multi-physics simulations with an emphasis on medical simulation. The main advantage of SOFA is its modularity, which allows a prototype simulation to be quickly built based on existing models. The SOFA framework has been widely used in medical simulation, including simulation of brain shift in deep brain stimulation[24], heart contraction using an electromechanical model [25], and augmented reality for laparoscopic images [26].

Simulations in SOFA are structured as a graph (tree or direct acyclic graph). The simulation is composed of nodes separating the different parts of the simulation, with each node including components which build the system to be solved. Each node of the graph corresponds to a structure of the valve: the annulus, the leaflets, and the chordae. All mechanical structures are solved in a one matrix system. An implicit Euler method is used for numerical integration. With stiff interaction forces, an implicit approach allows the simulation to remain stable while using large time steps. A time step of $0.001 \mathrm{~s}$ allows the simulation to remain interactive, and a velocity damping (defined by a damping Rayleigh mass $r_{M}$ of 0.1 and a Rayleigh stiffness $r_{K}$ of 0.5 ) was added to improve the convergence.

The dynamic equation expressed using an implicit Euler scheme is written as

$$
\mathbf{M} \ddot{u}_{k+1}+\mathbf{B} \dot{u}_{k+1}+\mathbf{K} u_{k+1}=f\left(x_{k+1}\right)
$$




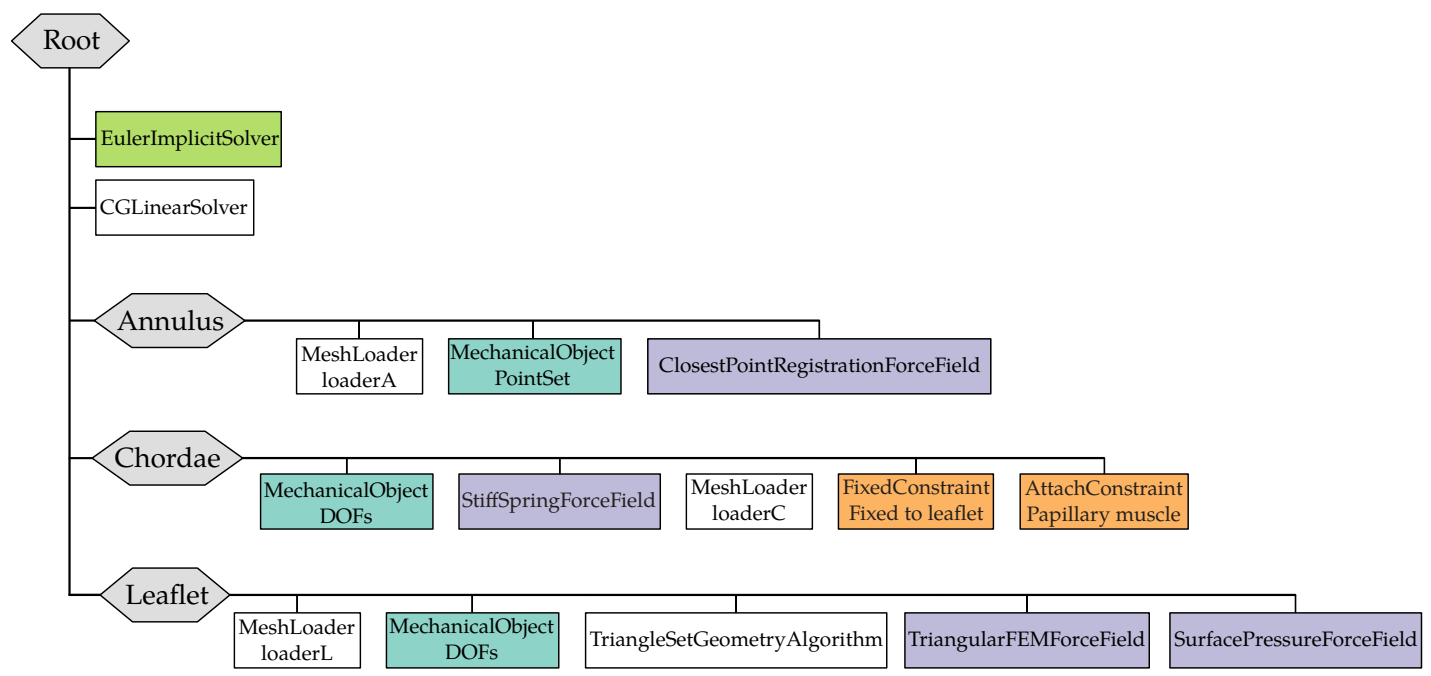

Figure 6. SOFA scene graph of the mitral valve closure simulation . Grey: geometrical nodes, White: Linear solver, Green Integration scheme, Purple: Forces, Orange: Constraints and Blue: Degrees of freedom of the body.

where $\mathbf{M}$ is the mass matrix, $\mathbf{u}$ is the displacement matrix, $\mathbf{K}$ is the stiffness matrix, $\mathbf{f}$ the external force matrix, $\mathbf{u}$ the displacement matrix and where the Rayleigh damping $\mathbf{B}$ equals:

$$
\mathbf{B}=r_{M} \mathbf{M}+r_{K} \mathbf{K}
$$

The leaflets were modeled as a surface of constant strain triangles with a linear elastic material model and constant thickness of $1 \mathrm{~mm}$. The corotational approach [27] was used since it supports large displacements. The SOFA implementation relies on a linear strain-stress relationship [28]. This corotational approach with linear elasticity is integrated in space using FEM. Poisson's ratio was set to 0.45 to model nearly incompressible tissue ([9]) and Young's modulus determination is described later in this paper. In addition, self-collision was monitored. For collision detection, an alarm distance was set slightly above the minimum edge length of the triangles. If a collision was detected and the point-to-surface distance was below a tenth of the alarm distance, a penalty force was applied. The spring stiffness of the contact force is one. Leaflet vertices near the annulus were pinned as in [7] to allow only rotation. For each constant strain triangle, the $9 \times 9$ stiffness matrix can be written as:

$$
\mathbf{K}_{t}=\mathbf{R}_{t}^{\top}\left\{\int_{S_{t}} \mathbf{B}_{t}^{\top} \mathbf{D}_{t} \mathbf{B}_{t} d S\right\} \mathbf{R}_{t}
$$

where $t$ describes a generic $\mathbf{P} 1$ triangular element, $\mathbf{B}_{t}$ is the strain-displacement matrix, $\mathbf{D}_{t}$ is the stress-strain matrix describing the mechanical constitutive law that is in plane stress condition and $\mathbf{R}_{t}$ is the rotation matrix.

Pressure due to blood is modeled as a surface-normal pressure on the leaflets. Fluid-structure interaction was not modeled as it would significantly increase computation time. Instead we applied a static pressure of $120 \mathrm{mmHg}$ to simulate typical peak systolic blood pressure. Under this pressure, the leaflets move from the initial state (unstressed) to a final state (pressurized). While we are interested only in the quasistatic equilibrium solution of the valve at peak systolic pressure, we 
solve for this state by solving the dynamic equations in the presence of collision detection and contact forces.

Given the annulus previously defined at the initial and final states of the valve (see §2.6), our strategy was to apply spring forces such that the annulus is pulled from its initial configuration toward its final shape at peak systole. The direction and intensity of the force for each vertex are given by the position of the closest point on the final state of the annulus. To recover the displacement of the annulus between the open and closed states of the valve, we used an iterative approach based on the iterative closest point method, implemented in SOFA [29, 30]. The points belonging to the annulus in the initial state were projected on the final state. The set of closest points (projection of the initial state onto the final state) was updated at each iterative step.

Chordae were defined by a stiff mass-spring system. The chordae material was modeled by an elastic law with parameters taken from [9]. The mass of the structures was lumped at discrete points, and, for an individual chord segment, we distributed its mass equally between its two endpoints. Three kinds of groups were created: point-masses inserted on the leaflet, point-masses attached at the papillary muscle, and other point-masses (branch points and flexure points). The points attached to the leaflet had their behavior determined by that of the associated leaflet vertex, the points attached to the papillary muscle assumed a zero-displacement Dirichlet condition, and the others had their motion defined by the mass-spring behavior of the chordae.

The complete simulation from the unstressed state to full closure at peak systole took 15 seconds. Simulations were run on a Macbook Air $2 \mathrm{GHz}$ Intel Core i7 with 8 GB of memory. The whole pipeline was scripted. The various steps associated with the average time on the three subjects are illustrated in Figure 7.

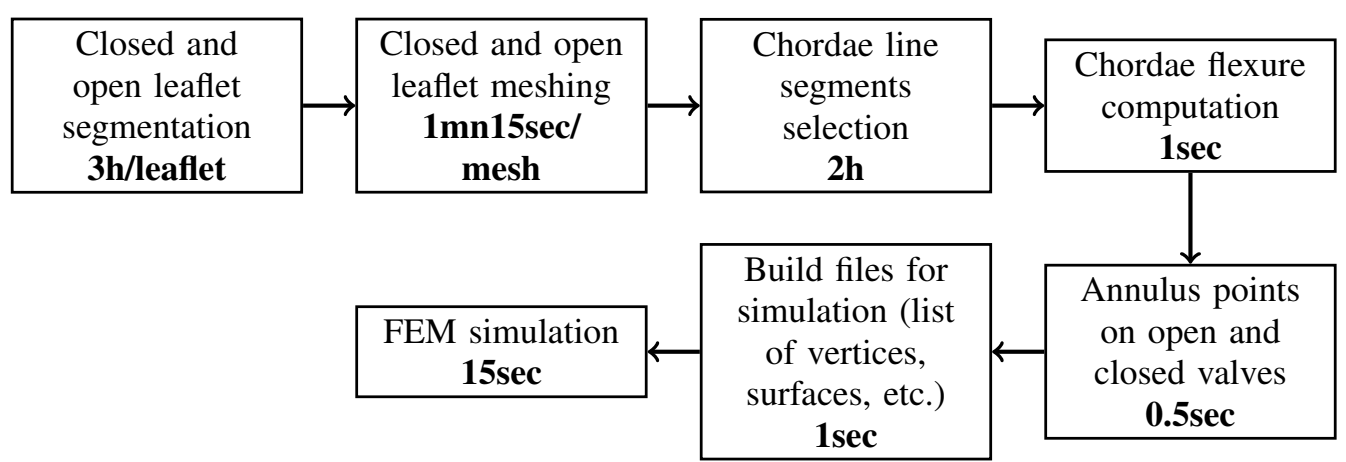

Figure 7. Simulation workflow with associated average time from the three subjects (in bold)

An illustration of the simulation is shown on Fig.8, and table II summarizes all the parameters involved in the simulation.

\section{RESULTS}

In this section we present how we applied our mitral valve computational model to test the different model parameters as well as the influence of the chordae tendineae structure. We define three metrics to evaluate our simulations. 

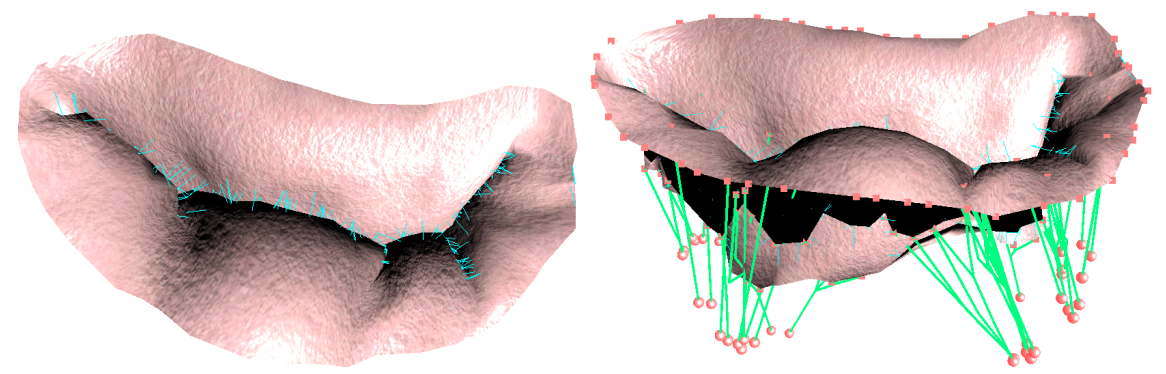

Figure 8. Valve rendering at the end of the simulation. Bump map rendering: leaflets, green: chordae, pink: attachment points and cyan: collision alarm distance .Left: Top view, right: lateral view.

Table II. Parameters of the mitral valve model

\begin{tabular}{|c|c|c|c|c|}
\hline Element & \multicolumn{2}{|c|}{ Geometrical parameters } & \multicolumn{2}{|c|}{ Biomechanical parameters } \\
\hline Leaflets & mesh & $\begin{array}{l}\approx 1500 \text { vertices } \\
\approx 2500 \text { triangles }\end{array}$ & $\begin{array}{l}\text { Young's Modulus } \\
\text { Poisson's ratio }\end{array}$ & $\begin{array}{c}\overline{[100,2500] \mathrm{kPa}} \\
0.45\end{array}$ \\
\hline Chordae & $\begin{array}{c}\text { mesh } \\
\text { attachment points }\end{array}$ & $\begin{array}{c}\approx 50 \text { line segments } \\
\approx 60 \text { with leaflet } \\
\approx 30 \text { with papillary muscles }\end{array}$ & Young's modulus & {$[20,40] \mathrm{MPa}$} \\
\hline Annulus & key positions & $\begin{array}{l}\approx 50 \text { initial positions } \\
\approx 50 \text { final positions }\end{array}$ & spring stiffness & $5000 \mathrm{~Pa}$ \\
\hline
\end{tabular}

\subsection{Metrics}

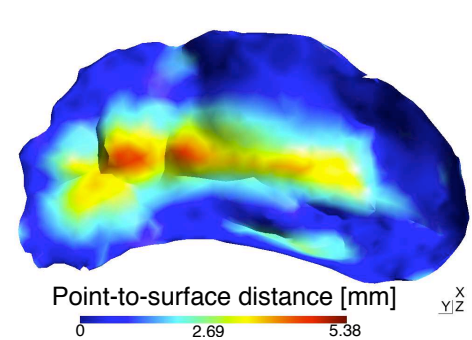

a) Error map

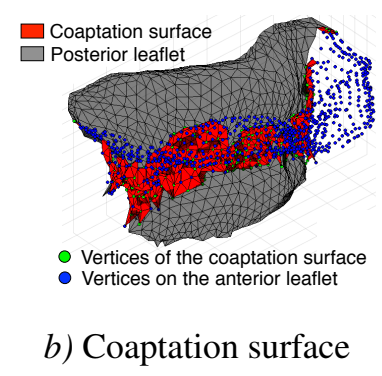

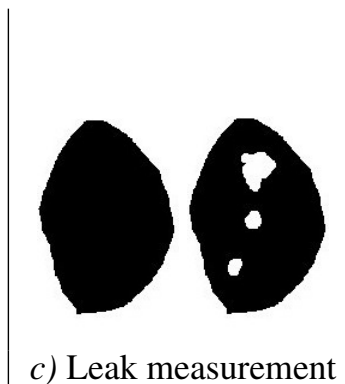

Figure 9. Metrics used to evaluate simulations. a) Error map with point-to-surface distance $b$ ) Area of coaptation surface $c$ ) Occluding contour, used as an indicator of leak.

For the first metric, we compute the mean distance between our simulated closed leaflets and the closed state extracted from the CT scan (ground truth) using an error-to-surface measurement. Specifically, from each point of the reference we compute the orthogonal projection on the simulation mesh. An illustration of this metric is given in Fig.9.a). The second metric that we use to assess the simulations is the area of interleaflet coaptation. The leaflet coaptation zone is critical to valve competency, and the depth and length of coaptation is viewed as an important indicator of mitral valve function [1]. For instance, in [31] the coaptation height is measured by intersecting the mesh with a plane that passes through the central coaptation axis and the midpoint of the leaflets attachment. In our case we measure the coaptation surface area by first separating the open leaflet mesh into faces belonging to the anterior leaflet and faces belonging to the posterior leaflet. Then a point-to-surface measurement is computed similarly to the previous metric between points of the 
anterior leaflet and triangles of the posterior leaflet. When their distance is less than one-tenth of the alarm distance (the contact distance defined in $\S 2.7$ ), the points are treated as belonging to the coaptation surface, and the area is computed. Fig.9.b) illustrates the coaptation surface computed in this way. The third metric for assessing simulations is a Boolean parameter that describes the absence or presence of a leak at peak systole. This metric is computed by extracting the occluding contour of the simulated leaflets from the $Z$ direction. An occluding contour corresponds to a boundary in the image between a 3D surface and a plane that it partially occludes. Fig.9.c).left shows an occluding contour of fully closed leaflets and Fig.9.c).right shows an occluding contour of leaflets that exhibit leak.

\subsection{Young's modulus estimation}

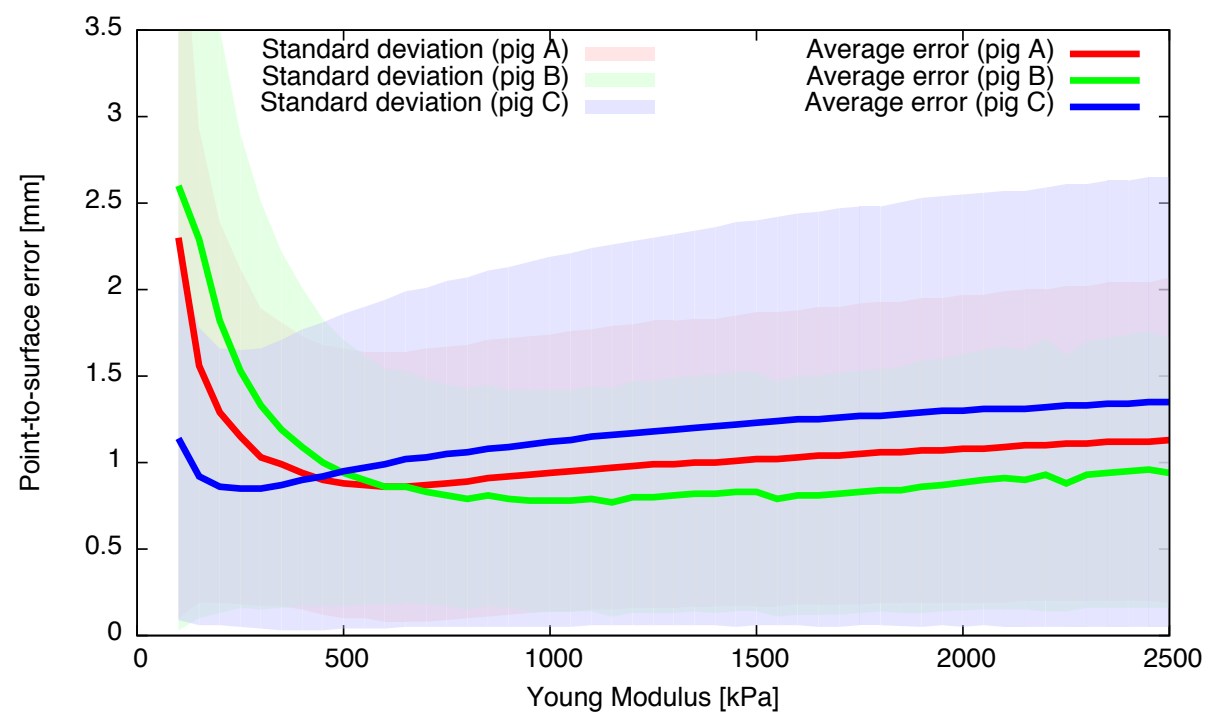

Figure 10. Point-to-surface distance error as a function of Young's modulus.

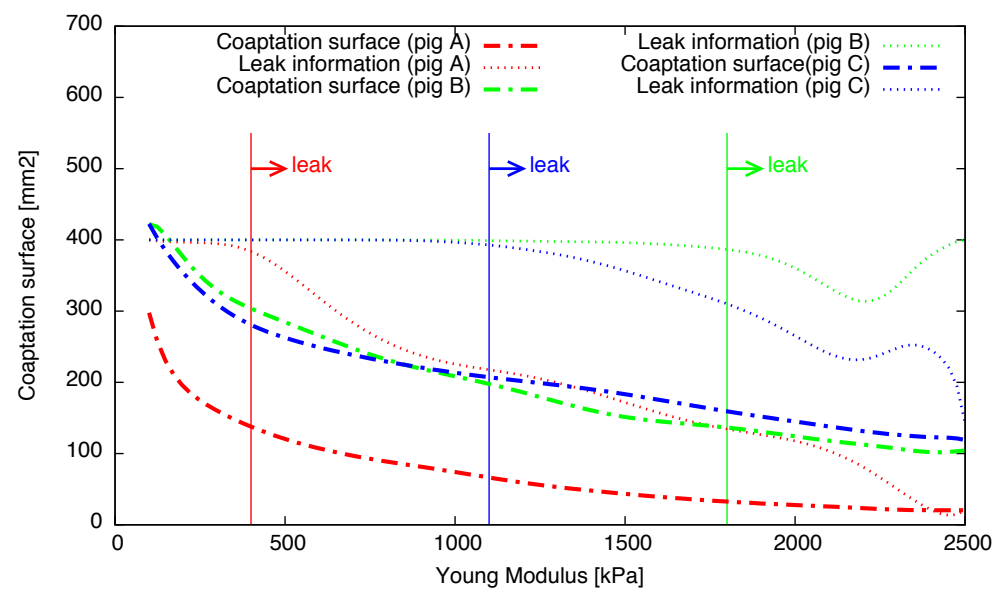

Figure 11. Coaptation surface and leak information as functions of Young's modulus. 
The first model parameter that we estimate is Young's modulus. While most of the parameters can be determined from direct measurement (e.g. pressure) or from basic principles (e.g. Poisson's ratio), leaflet elasticity is known to vary among individuals and must be uniquely determined for each subject. To estimate this modulus for each case, we ran simulations while varying this parameter over a large range of values, and the evaluation metrics for each simulation were compared to those from the in vivo data. For each value of Young's modulus, we compute i) the point-tosurface distance between the simulated closed valve surface at peak systole and the actual closed valve surface from the micro CT scan, ii) the coaptation surface area, and iii) the area of the occluding contour. Results for our three pig data sets are shown in Fig. 10 and 11. Results show that underestimating the leaflet elasticity can lead to significant error, which is due to bulging of the leaflets between the annulus and chordae attachment points. Underestimating leaflet elasticity also causes the coaptation surface to be stretched beyond its actual size and tends to underestimate the area of the occluding contour, which underestimates the presence of leak. This is illustrated in Fig. 12. The vertical lines in Fig. 11 indicate the value of Young's modulus at which the leak metric starts to detect a leak. The associated curve is a plot of normalized shadow surface area (which decreases as the gaps that allow light to pass grow). The dotted line in Fig. 12 representing the bulged leaflets is far from the actual surface while still having a larger coaptation surface. By comparison, an over-estimation of Young's modulus only produces a slight increase in error. This can be explained by the fact that the leaflet does not stretch enough to prevent leaks. The error does not increase dramatically because, as illustrated in Fig. 12 by the dashed line, the leaflets simply tighten.

The optimal Young's modulus for all three pig subjects is approximately $400 \mathrm{kPa}$. It is the lowest Young's modulus value that works on all the subjects. The simulation of valve closing without anisotropy implies a very elastic material as explained above. $400 \mathrm{kPa}$ is a value that can be found at the drop in occluding contour area for pig A on Fig. 11 and the relative constancy of point-to-surface error to the right of $400 \mathrm{kPa}$ on Fig. 10. The disparity among subjects is due to leaflet thickness, which is not the same for the three pigs. Disparity in leaflet thickness among subjects is probably responsible for part of this disparity, but the normal variability seen in heart valve leaflet properties is huge and is probably a major contributor. It is described in the analysis on mechanical properties for mitral leaflets in [32]. The minimum error is approximately $1 \mathrm{~mm}$, which is comparable to other results from the literature. The standard deviation is around $0.7 \mathrm{~mm}$, which shows that the error is sufficiently uniform.

Our estimated value of the Young's Modulus is low compared to published values (e.g. : $E_{1} \sim$ $2000 \mathrm{kPa}$ and $E_{2} \sim 6000 \mathrm{kPa}$ in the linear anisotropic model from [9]). This is due to our assumptions of a linear constitutive law and isotropic behavior. The mitral valve leaflets have been shown to exhibit a highly nonlinear stress-strain relationship, with very low in-plane stiffness at low strains transitioning to much higher stiffness at high strains, and published biaxial testing curves show two distinct regimes: a nearly linear low stiffness regime at low stresses followed by a transition to a high stiffness regime at high stresses.

With our limitation of a linear stress-strain relationship, it was necessary to model the low stiffness regime in order to capture the considerable tissue stretch observed in the actual valve as the valve begins to close. If the leaflet tissue were modeled with a single stiffness (linear stress-strain relationship passing through the origin) corresponding to the slope of the high stiffness regime, 
the leaflet would undergo no appreciable strain leading to little to no interleaflet coaptation, tethered leaflets, and underestimation of the occluding contour. In order to simulate the high strain undergone by leaflet tissue as they begin to be loaded, which is critical to normal valve function, we found it necessary to use the Young's modulus value corresponding to the low stiffness regime.

In addition to leaflet tissue nonlinearity, the leaflets are also anisotropic, exhibiting greater inplane stiffness in the predominant direction of the collagen network within the leaflets. Again, in order to capture the high-strain behavior of the leaflets, we used a low Young's modulus more typical of the material direction perpendicular to the predominant collagen direction.

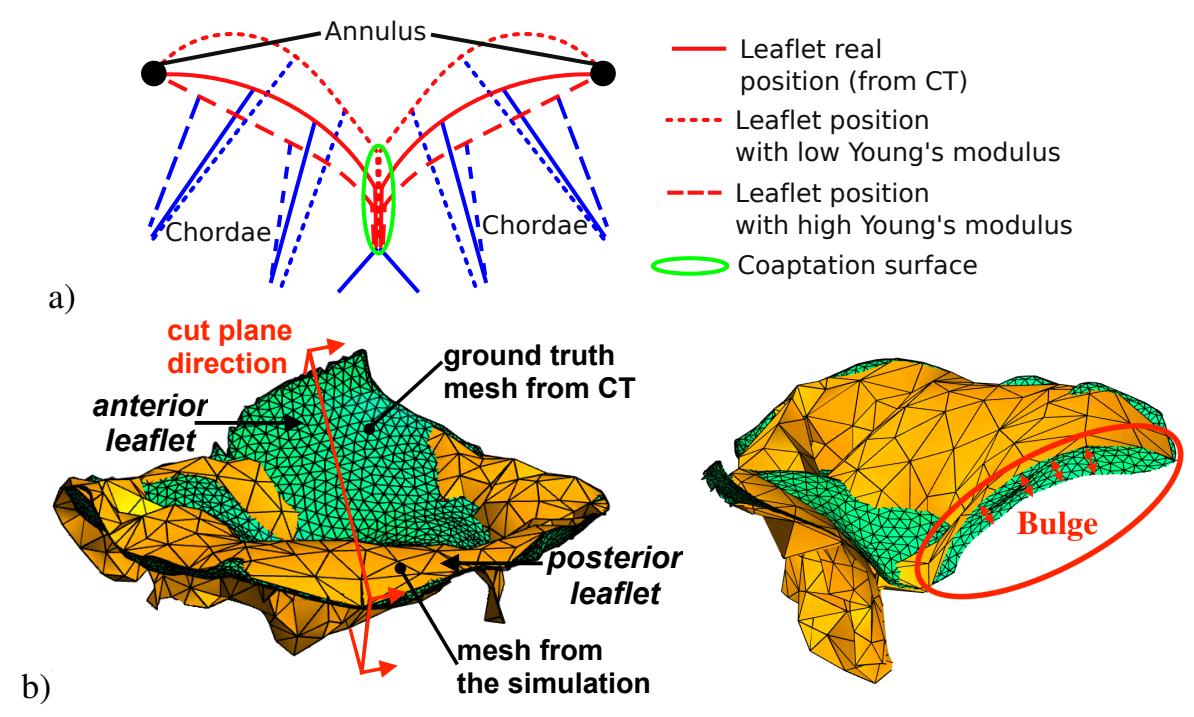

Figure 12. Comparison between simulation mesh and ground truth: a) Illustration of the leaflet behavior while linearly increasing Young's modulus. b) Example showing the bulging effect in a cut plane through the posterior and anterior leaflets

Valve closure has been simulated at $120 \mathrm{mmHg}$ and $150 \mathrm{mmHg}$ pressures (see Fig.13). Closure in both cases is maintained and chordae are outstretched in the same way.

Table III shows how the chordae behave during stretching with the two pressures. We have computed the stretch ratio $S_{t r} R\left(P_{\text {press }}\right)$ at a pressure $P_{\text {press }=1}=120 \mathrm{mmHg}$ and $P_{\text {press }=2}=150$ mmHg with: $S_{t r} R\left(P_{\text {press }}\right)=\frac{\text { chord length at } P_{\text {press }}}{\text { initial chord length }}$ then computed mean and std across all chordae segments for each of the 2 pressures. Stretch ratio is approximately 1.003 for both pressures so there is no appreciable stretch.

Table III. Stretch ratio on 104 chordae in pig A with $120 \mathrm{mmHg}$ and $150 \mathrm{mmHg}$ pressures

\begin{tabular}{|c|c|c|}
\hline Pressure & average & standard deviation \\
\hline $120 \mathrm{mmHg}$ & 1.0031 & 0.0027 \\
\hline $150 \mathrm{mmHg}$ & 1.0035 & 0.0030 \\
\hline
\end{tabular}




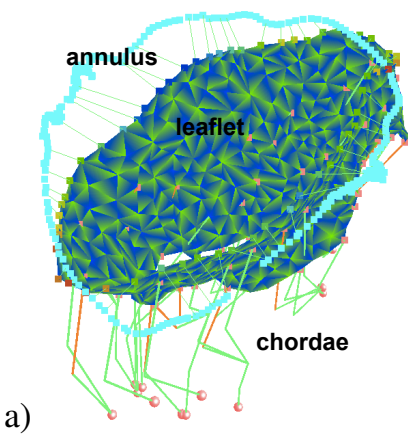

b)

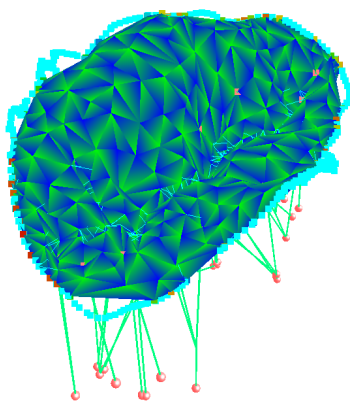

c)

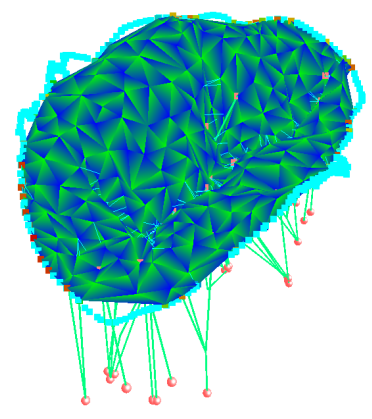

Figure 13. Influence of the pressure on the chordae: a) no pressure, b) $120 \mathrm{mmHg}$ pressure and c) $150 \mathrm{mmHg}$ pressure.

\subsection{Sensitivity to mesh resolution}

Next, we assess the effect of varying the mesh resolution on simulation accuracy. We generated meshes with increased resolution using the method of mid-point triangle subdivision, and we evaluated results with the number of subdivisions $n_{\text {sub }}=0, n_{\text {sub }}=1$ and $n_{\text {sub }}=2$. Fig. 14. left shows a plot similar to Fig. 10. The error evolution has been computed between simulations using the finest mesh $\left(n_{s u b}=2\right)$ and simulations using the two coarser meshes $\left(n_{\text {sub }}=0\right.$ and $\left.n_{\text {sub }}=1\right)$. As previously, the simulation is performed with various Young's moduli. Only the point-to-surface error and its standard deviation are plotted, and only one porcine valve is shown to avoid information redundancy (pig $A$ ).

There is little difference between the errors (less than $0.3 \mathrm{~mm}$ for Young's modulus greater than $E=500 \mathrm{kPa}$ ), and the coarsest mesh appears sufficient. The differences among the results for the three subdivisions are mainly due to the errors around the annulus. Fig.14.right shows problems that can occur around the annulus when the boundary is not discretized finely enough to match the geometry. However, this phenomena occurs at a relatively small number of points and has little impact on the simulated behavior of the valve.
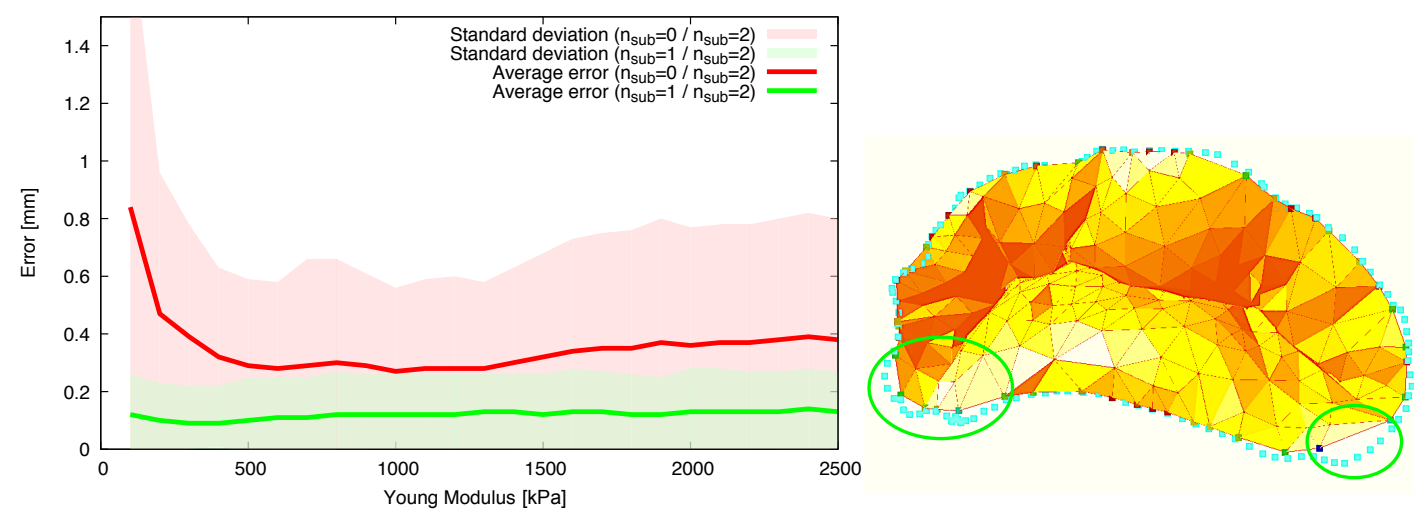

Figure 14. Mesh subdivision sensitivity. Left: point-to-surface distance error with various Young's moduli, right: Mesh area where more vertices are necessary. 


\subsection{Rigid registration estimation}

Another important degree of freedom of our model is the initial rigid body motion. The pressurized and unpressurized states of the mitral valve were taken from micro CT imaging, and in general the position within the scanner for the two images was different. While the rotations as well as the horizontal translations $(X$ and $Y$ ) were correct because the annulus is well centered, the translation in $Z$ was less certain. In order to estimate it, we added to our model a $Z$ translation of the final state of the annulus for the iterative closest point force field (as described in §2.7). Because this will also change the way the leaflets are tightening (Fig. 12), we needed to also optimize the Young's modulus value.

The optimization of these two parameters was done using the pattern search algorithm [33]. The initial values of the parameters were relatively close to their optimal: Young's modulus was chosen from the analysis in Figure 11, and the heart position in the CT scanner did not differ much between the two experimental conditions. The objective function used for the optimization was the mean point-to-surface error measurement. We chose point-to-point distance because the computation time is significantly less than for the point-to-surface error metric used in $\S 3.1$. The aim here is not to validate a simulation but to evaluate the influence of the rigid registration on the simulation. An important advantage to this metric is that it does not require computation of the gradient. Results are presented in Fig. 15. They show that the $Z$ translation of the rigid registration computed with the iterative closest point algorithm already gives a satisfactory result. The error is slightly greater than before because we previously used the point-to-point distance to decrease computation time.

The vertical displacement of the rigid registration between the two micro CT scans is less than the average point-to-surface error. This means that the error is not due to poor registration of the two micro CT scans but from limitations of our method.
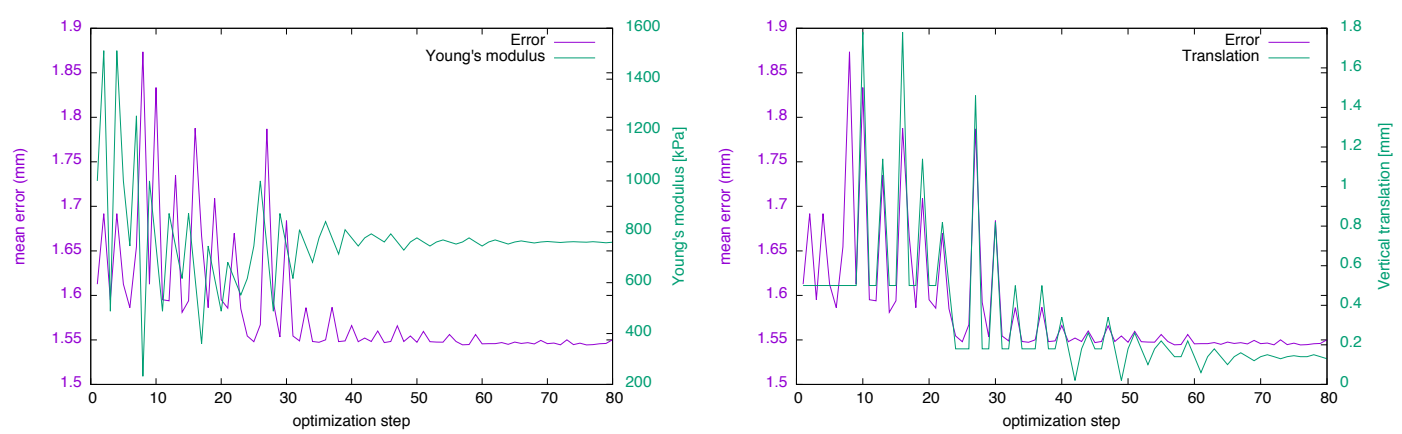

Figure 15. Error variation while optimizing Young's modulus and the vertical translation of the rigid transform.

\subsection{Influence of the attachment point}

Having assessed model robustness and quantified the sensitivity of the simulated valve shape to changes in model parameters, we applied it to real case studies. Our primary focus was on the influence of chordae on the simulation. In particular, we focused on the influence of the chordae attachment points. Anatomically there are three main types of chordae: marginal (primary) chordae, intermediate (secondary) chordae, and basal (tertiary) chordae. Since the last of these are attached 
near the annulus, they are not considered in our analysis. Marginal and intermediate chordae can be seen in Fig. 16.left, which shows the valve model for one of the pig subjects.

We first assessed the influence of the chordae by computing our metrics with: i) all chordae, ii) no chordae, iii) no intermediate chordae and iv) no marginal chordae (Fig. 16.right). The only configuration where the valve closed normally was with all chordae, and, as expected, removing all chordae allowed the leaflets to prolapse in the $Z$ direction. The marginal chordae had a greater influence on the closed valve: the error and standard deviation were higher with no marginal than with no intermediate chordae. To assess the influence of each chord as well as the importance of its attachment position, we ran a leave-one-out test. The result is displayed in Fig. 17. The high rate of absence of leak as well as the mean point-to-surface error shows that the chordae are robust to rupture of a single chordae even if the coaptation surface significantly changes. However, in the two cases of very small coaptation surface area, a leak was well detected. The coaptation surface area when removing marginal chordae was smaller than when removing intermediate chordae. This shows the contribution of the marginal chordae on pulling down the leaflet to maximize coaptation.
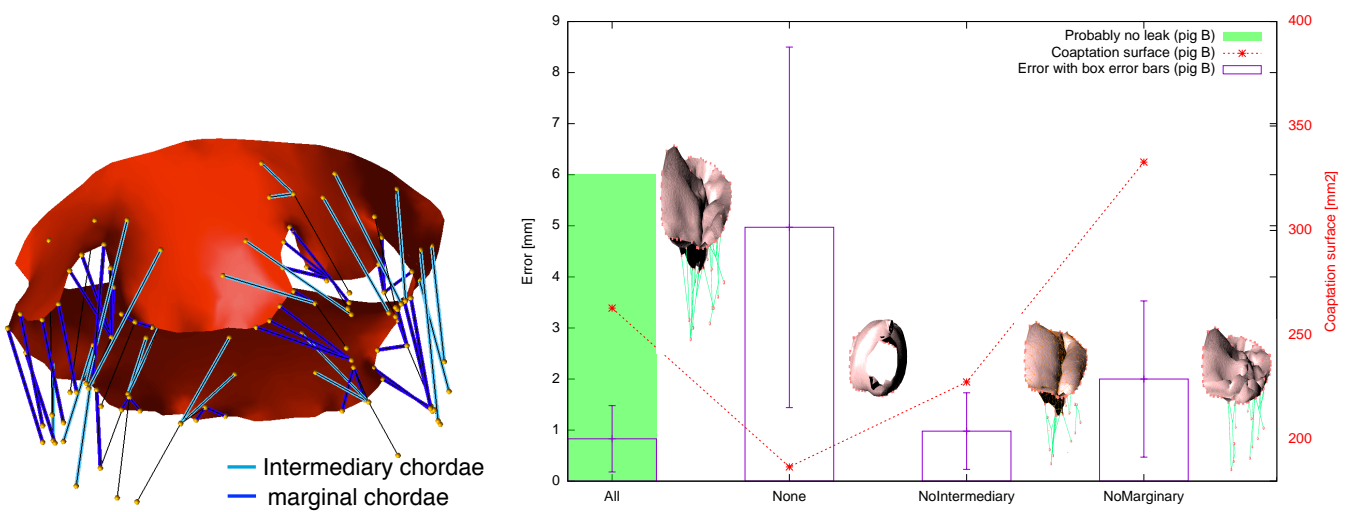

Figure 16. Chordae influence. Left:attachment point labeling, right: Metric plots based on different chordae configurations.
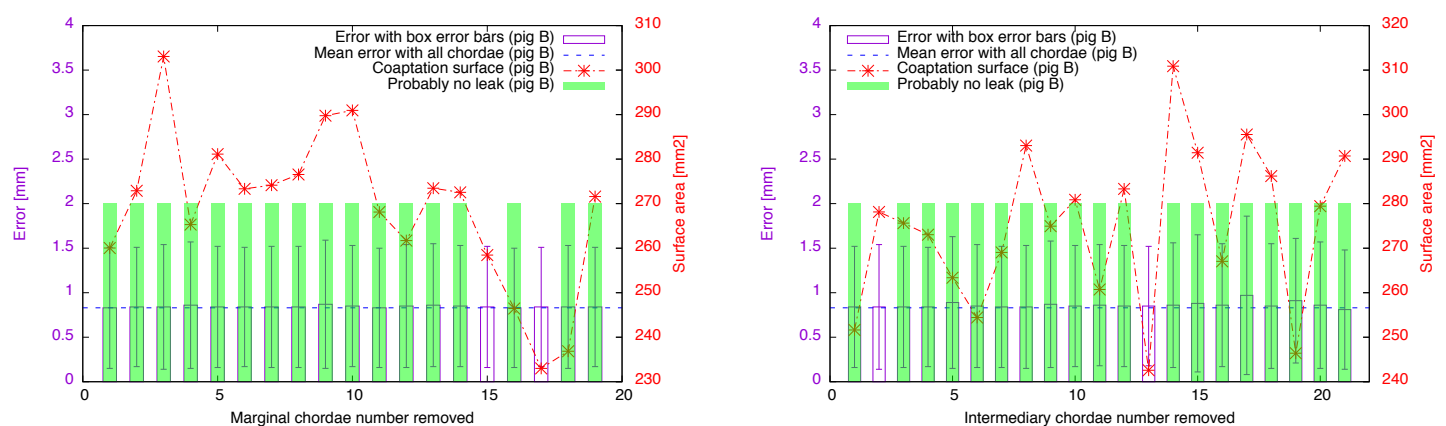

Figure 17. Error metric evolution with the leave-one-out method for each chord. Chordae are separated into categories marginal and intermediate chordae.

Furthermore, we implemented the measure of the coaptation surface as the coming together of either atrial or ventricular surfaces of the leaflets. An explanation of this behaviors is that the contact surface area was slightly higher without marginal chordae than with all chordae because i) the 
intermediate chordae constrain the two leaflets to remain close enough so the valve is not wide open like in the case of no chordae, and ii) the contact forces allow the two leaflets to remain in contact while being pushed upward. However, the coaptation surface was not continuous, which is why holes (and thus leak) appeared

The coaptation area evolution graph shows some behavior that is not coherent. Specifically, coaptation area is slightly higher without marginal chordae than with all chordae. This is due to the intermediate chordae constraining the two leaflets to remain close together while the contact forces and small but not insignificant bending stiffness prevent the leaflets from buckling and prolapsing, as would happen in the the real valve. However, even though there is substatial coaptation area in this case, the coaptation area is not continuous - which is why holes (and thus leak) appear.

\subsection{Limitations}

Our methodology shows promising results such as fast computation time, the possibility to model valve closure in a subject-specific capacity, and the utility of robust metrics for quantifying valve simulation. However we acknowledge drawbacks to our framework.

The unstressed leaflets were directly extracted from the micro CT scan. One of the two 3D images we scanned was a valve resting downward, without any pressure. Residual stresses that have been shown to exist between layers of the multilayered valve leaflet tissue have been neglected and the leaflet tissue was treated as a homogeneous material.

The change in configuration of the annulus between the open and closed states of the valve was extracted from the subject data. The deformation was based on spring forces that drive the annulus from the open to the closed valve configuration. Systole was experimentally modeled by passively inflating the heart, corresponding to the annulus undergoing passive stretch. However, in vivo, systole is accompanied by contraction of the annulus and reduction in its size.

The value of Young's modulus that allowed the valve to close is lower than physiological values. If a realistic value is used, the leaflet motion is restricted. It is likely that adopting a nonlinear anisotropic constitutive model would permit normal motion because it would permit greater leaflet strain in the direction of the valve center. It also influences the coaptation surface metric measurement.

The effects of our assumptions of uniform leaflet thickness and uniform chordae diameters have not been assessed. Both features are known to be nonuniform, and neglecting to model this may have impacted simulation results.

This analysis has been performed using micro CT because its high resolution enabled us to resolve very fine valve structures. While micro CT cannot be used clinically, we are investigating methods to segment chordae from clinical 3D ultrasound based on previous work from our group [34]. We are also exploring the use of 4D cardiac CT scans.

Finally, our framework focused on the simulation of a static closed valve at peak systole, but it might be informative to simulate a time-dependent pressure during valve closure. However, while the quasistatic nature of our simulations is a limitation, there is considerably clinical value to using simulation to predict valve competence during peak systole because a mitral valve that is competent at peak systole is unlikely to exhibit clinically significant regurgitation.

We will address these model limitations in future work. 


\section{CONCLUSION}

In this article, we have presented a method to create a image-based computational model of the mitral valve based on volumetric images. The contribution of this paper are: i) the geometry of the valve model, which is more accurate than current simple parametric models that are widely used in the literature, ii) the automatic computation of the full valve structure, including the leaflets, annulus, chordae, and boundary conditions (annulus displacement, chordae fixed points on papillary muscles, chordae-leaflet attachments, and chordae flexure). Our framework requires only minor input from the user to prepare the biomechanical model, which is particularly useful where there are many subjects to test. It also allows quick variation of the input data (e.g.: changing the mesh resolution or the annulus displacement), iii) the fast FEM implementation within SOFA, which is robust (tested by many simulations on a cluster), runs in interactive-time (the complete closing process takes 15 seconds), and is accurate (the error compared to real data is around $1 \mathrm{~mm}$ ), iv) the development of three metrics to evaluate a mitral valve closure simulation (a point-to-surface error measurement with a ground truth reference extracted from the CT image, leaflet coaptation surface area, and an indication if the simulated closed leaflets leak.), and v) the quantitative comparison of the influence of marginal versus intermediate chordae on the simulation.

Results from FEM analysis on three explanted porcine hearts show that it is possible to predict the shape of the leaflets at peak systole with an average surface error of approximately $1 \mathrm{~mm}$. We also measure the influence of the positions of the chordae tendineae on simulation results and show that marginal chordae have a greater influence on the final shape than intermediate chordae.

In future work, we plan to use an anisotropic hyperelastic law to model the leaflet behavior in order to increase accuracy by having both an image-based collagen fiber orientation and a nonlinear constitutive law to model the increasing leaflet stiffness with increasing leaflet strain. We also plan to test our model on more data. This is important to demonstrate model robustness for a system with so many degrees of freedom and such large ranges of physiological parameters. Finally, we want to continue evaluating the role of the chordae by studying the influence of their diameters.

\section{REFERENCES}

1. Carpentier A, Adams D, Filsoufi F. Carpentier's Reconstructive Valve Surgery E-Book. Elsevier Health Sciences, 2010.

2. Kunzelman K, Einstein D, Cochran R. Fluid-structure interaction models of the mitral valve: function in normal and pathological states. Philosophical Transactions of the Royal Society of London B: Biological Sciences 2007; 362(1484):1393-1406, doi:10.1098/rstb.2007.2123.

3. Burlina P, Sprouse C, DeMenthon D, Jorstad A, Juang R, Contijoch F, Abraham T, Yuh D, McVeigh E. Patientspecific modeling and analysis of the mitral valve using 3d-tee. Information Processing in Computer-Assisted Interventions. Springer, 2010; 135-146.

4. Wenk JF, Zhang Z, Cheng G, Malhotra D, Acevedo-Bolton G, Burger M, Suzuki T, Saloner DA, Wallace AW, Guccione JM, et al.. First finite element model of the left ventricle with mitral valve: Insights into ischemic mitral regurgitation. The Annals of Thoracic Surgery 2010; 89(5):1546 - 1553, doi:http://dx.doi.org/ 10.1016/j.athoracsur.2010.02.036. URL http://www.sciencedirect.com/science/article/pii/ S0003497510004121.

5. Sprouse C, Mukherjee R, Burlina P. Mitral valve closure prediction with 3-d personalized anatomical models and anisotropic hyperelastic tissue assumptions. IEEE Trans. Biomed. Engineering 2013; 60(11):3238-3247, doi: 10.1109/TBME.2013.2272075. URL http: / / dx. doi .org/10.1109/TBME.2013.2272075. 
6. Wang Q, Sun W. Finite element modeling of mitral valve dynamic deformation using patient-specific multislices computed tomography scans. Annals of Biomedical Engineering 2013; 41(1):142-153, doi:10.1007/ s10439-012-0620-6. URL http://dx.doi.org/10.1007/s10439-012-0620-6.

7. Kunzelman KS, Cochran RP, Verrier ED, Eberhart RC. Anatomic basis for mitral valve modelling. J Heart Valve Dis Sep 1994; 3(5):491-496.

8. Kunzelman KS, Reimink MS, Cochran RP. Annular dilatation increases stress in the mitral valve and delays coaptation: A finite element computer model. Vascular 1997; 5(4):427-434, doi:10.1177/096721099700500416.

9. Cochran RP, Kunzelman KS. Effect of papillary muscle position on mitral valve function: relationship to homografts. The Annals of Thoracic Surgery 2014/12/26; 66(6):S155-S161, doi:10.1016/S0003-4975(98)01100-X. URL http: //www. annalsthoracicsurgery.org/article/S0003-4975(98)01100-X/abstract.

10. Lee $\mathrm{C}$, et al. . A high-fidelity and micro-anatomically accurate $3 \mathrm{~d}$ finite element model for simulations of functional mitral valve. Functional Imaging and Modeling of the Heart, LNCS, vol. 7945. Springer, 2013; 416-424, doi: 10.1007/978-3-642-38899-6_49. URL http://dx.doi.org/10.1007/978-3-642-38899-6_49.

11. Gorman J, et al.. Dynamic three-dimensional imaging of the mitral valve and left ventricle by rapid sonomicrometry array localization. The Journal of Thoracic and Cardiovascular Surgery 2014/12/31; 112(3):712-724, doi: 10.1016/S0022-5223(96)70056-9. URL http://www. jtcvsonline.org/article/s0022-5223 (96) $70056-9 /$ abstract.

12. Prot V, Haaverstad R, Skallerud B. Finite element analysis of the mitral apparatus: annulus shape effect and chordal force distribution. Biomechanics and Modeling in Mechanobiology 2009; 8(1):43-55, doi:10.1007/ s10237-007-0116-8. URL http://dx.doi .org/10.1007/s10237-007-0116-8.

13. Holzapfel GA, Sommer G, Gasser CT, Regitnig P. Determination of layer-specific mechanical properties of human coronary arteries with nonatherosclerotic intimal thickening and related constitutive modeling. American Journal of Physiology - Heart and Circulatory Physiology 2005; 289(5):H2048-H2058, doi:10.1152/ajpheart.00934.2004.

14. Hammer P, del Nido P, Howe R. Anisotropic mass-spring method accurately simulates mitral valve closure from image-based models. Functional Imaging and Modeling of the Heart, Lecture Notes in Computer Science, vol. 6666, Metaxas D, Axel L (eds.). Springer Berlin Heidelberg, 2011; 233-240, doi:10.1007/978-3-642-21028-0_29. URL http://dx.doi.org/10.1007/978-3-642-21028-0_29.

15. Tenenholtz NA, Hammer PE, Schneider RJ, Vasilyev NV, Howe R. On the design of an interactive, patient-specific surgical simulator for mitral valve repair. Intelligent Robots and Systems (IROS), 2011 IEEE/RSJ International Conference on, 2011; 1327-1332, doi:10.1109/IROS.2011.6095089.

16. Toma M, Jensen MØ, Einstein DR, Yoganathan AP, Cochran RP, Kunzelman KS. Fluid-structure interaction analysis of papillary muscle forces using a comprehensive mitral valve model with $3 \mathrm{~d}$ chordal structure. Annals of Biomedical Engineering 2015; .

17. Yushkevich PA, Piven J, Cody Hazlett H, Gimpel Smith R, Ho S, Gee JC, Gerig G. User-guided 3D active contour segmentation of anatomical structures: Significantly improved efficiency and reliability. Neuroimage 2006; 31(3): 1116-1128.

18. Zhu SC, Yuille A. Region competition: Unifying snakes, region growing, and bayes/mdl for multi-band image segmentation. IEEE Transactions on Pattern Analysis and Machine Intelligence 1996; 18:884-900.

19. Caselles V, Catté F, Coll T, Dibos F. A geometric model for active contours in image processing. Numerische Mathematik 1993; 66(1):1-31, doi:10.1007/bf01385685. URL http: / / dx . doi .org/10.1007/ bf 01385685 .

20. Rosenfeld A, Pfaltz JL. Sequential operations in digital picture processing. J. ACM Oct 1966; 13(4):471-494, doi: 10.1145/321356.321357. URL http: / / doi . acm.org/10.1145/321356.321357.

21. Censi A. An ICP variant using a point-to-line metric. Proceedings of the IEEE International Conference on Robotics and Automation (ICRA), Pasadena, CA, 2008, doi:10.1109/ROBOT.2008.4543181. URL http://purl.org/ censi/2007/plicp.

22. Lévy B, Liu Y. Lp centroidal voronoi tessellation and its applications. ACM Transactions on Graphics (TOG) 2010; 29(4): 119.

23. Faure F, Duriez C, Delingette H, Allard J, Gilles B, Marchesseau S, Talbot H, Courtecuisse H, Bousquet G, Peterlik I, et al.. SOFA: A Multi-Model Framework for Interactive Physical Simulation. Soft Tissue Biomechanical Modeling for Computer Assisted Surgery, Studies in Mechanobiology, Tissue Engineering and Biomaterials, vol. 11, Payan Y (ed.). Springer, 2012; 283-321, doi:10.1007/8415 \_2012\_125. URL https://hal.inria. fr/hal-00681539.

24. Bilger A, Bardinet E, Fernández-Vidal S, Duriez C, Jannin P, Cotin S. Intra-operative Registration for Deep Brain Stimulation Procedures based on a Full Physics Head Model. MICCAI 2014 Workshop on Deep Brain Stimulation Methodological Challenges - 2nd edition, Boston, United States, 2014. URL https://hal.inria.fr/ hal-01060304. 
25. Talbot H, Marchesseau S, Duriez C, Courtecuisse H, Relan J, Sermesant M, Cotin S, Delingette H. Interactive Electromechanical Model of the Heart for Patient-Specific Therapy Planning and Training using SOFA. VPH 2012, Londres, United Kingdom, 2012. URL https: //hal.inria.fr/hal-0 0751537.

26. Haouchine N, Dequidt J, Peterlik I, Kerrien E, Berger MO, Cotin S. Image-guided Simulation of Heterogeneous Tissue Deformation For Augmented Reality during Hepatic Surgery. ISMAR - IEEE International Symposium on Mixed and Augmented Reality 2013, Adelaide, Australia, 2013. URL https://hal.inria.fr/ hal-00842855.

27. Felippa C, Haugen B. A unified formulation of small-strain corotational finite elements: I. theory. Computer Methods in Applied Mechanics and Engineering 2005; 194(21-24):2285 - 2335, doi:http:// dx.doi.org/10.1016/j.cma.2004.07.035. URL http://www.sciencedirect.com/science/article/ pii/S 0045782504005353 , computational Methods for Shells.

28. Nesme M, Payan Y, Faure F. Efficient, physically plausible finite elements. Eurographics (short papers), Dingliana J, Ganovelli F (eds.), 2005. URL http: / / www-evasion.imag.fr/Publications/2005/NPF0 5.

29. Rusinkiewicz S, Levoy M. Efficient variants of the ICP algorithm. Third International Conference on $3 D$ Digital Imaging and Modeling (3DIM), 2001.

30. Simon D. Fast and accurate shape-based registration. PhD Thesis, Robotics Institute, Carnegie Mellon University, Pittsburgh, PA December 1996.

31. Hammer PE, Pacak CA, Howe RD, del Nido PJ. Collagen bundle orientation explains aortic valve leaflet coaptation. Proceedings of the 7th International Conference on Functional Imaging and Modeling of the Heart, FIMH'13, Springer-Verlag: Berlin, Heidelberg, 2013; 409-415, doi:10.1007/978-3-642-38899-6_48. URL http: //dx.doi.org/10.1007/978-3-642-38899-6_48.

32. Krishnamurthy G, Ennis DB, Itoh A, Bothe W, Swanson JC, Karlsson M, Kuhl E, Miller DC, Ingels Jr NB. Material properties of the ovine mitral valve anterior leaflet in vivo from inverse finite element analysis. Am J Physiol Heart Circ Physiol 2008; 295(3):H1141-H1149.

33. Hooke R, Jeeves TA. “ direct search” solution of numerical and statistical problems. J. ACM Apr 1961; 8(2):212229, doi:10.1145/321062.321069. URL http://doi.acm.org/10.1145/321062.321069.

34. Schneider RJ, Perrin DP, Vasilyev NV, Marx GR, del Nido PJ, Howe RD. Mitral annulus segmentation from 3d ultrasound using graph cuts. IEEE transactions on medical imaging 2010; 29 9:1676-87. 